\title{
Tartrate Resistant Acid Phosphatase Staining Method
}

National Cancer Institute

\section{Source}

National Cancer Institute. Tartrate Resistant Acid Phosphatase Staining Method. NCI

Thesaurus. Code C118401.

A staining method used to demonstrate the presence of acid phosphatase and tartrate resistant acid phosphatase (TRAP) in osteoclasts. 